\title{
Data Transfer among Urology Research Centers in Universities of Medical Sciences
}

\author{
Abdolhossein Ayoubi* ${ }^{1}$, Mohammad Sadegh Sanie ${ }^{2}$, Moharram Kazemi ${ }^{3}$ \\ and Saman Sobh Heydari ${ }^{4}$ \\ ${ }^{1,3,4}$ M.Sc of Medical Informatics Technology, Biomedical Engineering \\ Department, Amirkabir University of Technology, Tehran, Iran \\ ${ }^{2} \mathrm{MD}$ in Anesthesia and critical care, Assistant Professor at Jahrom University of \\ Medical Sciences \\ ${ }^{1}$ ayoubi.hossein@gmail.com; ${ }_{4}^{2}$ Sadegh_532@yahoo.com; ${ }^{3}$ kazemilaz@yahoo.com,
}

\begin{abstract}
This paper studies Health Information System (HIS) for administrative automation by the use of library and internet resources. The Goal is to identify valid urology research centers in the country so that scientific communications be increased; indeed idea exchange, technology and up-to-date urology knowledge be achieved.Thus, we will be able to promote the science level and to search novel diagnostic methods and to treat urology diseases especially kidney disease. There have been some collaborations with executive-research centers in the country in order to do some researches in urology field of study [1]. The method applied here is making use of different data transfer styles to clarify which one is suitable for urology research centers. Finally, it can be declared as conclusion that an available health information system brings a better decision making in the treatment processes and develops therapeutic results. The contribution of this study is significant when management of Information is on the focus in health systems.
\end{abstract}

Keywords: Health Information System, Administrative Automation, Urology Research Center, Health Level Seven

\section{Introduction}

The goal of Information Health System in administrative automation is to support hospitalized activities in practical, technical and strategic level. In other words, the goal is to make use of computer software and hardware in supplying, saving, processing, reviewing, and communicating with administrative information in total hospital activities and to satisfy all addressees' needs. It is a fact that nowadays it is not possible any more to involve in traditional process of bedridden or outpatient records. Thus, in order to increase accuracy and to facilitate services to patients, accelerate establishing and rotation of the records in hospital, quick detection of records for various aims such as university students' research and studies and the possibility of facilitated use of medical documents. Establishing HIS seems to be essential in all hospitals [2]. HIS solves all the issues for a hospital and changes the hospital from a traditional hospital to a modern medical center. Of course, it should be noted that Hospital Information System is not just confined to medical issues. A hospital is an organization consisted of sections, clinical and paraclinical unites which are organized with support units as a unified unit while the whole collection seeks a determined goal. Support unites are known as MIS, and they are linked to medical unites while all their activities are along presenting services to patients. Therefore, treatment and support are considered supplementary, in other words, a combination of HIS and MIS establishes a comprehensive system, HIMS, which is also 
called HMIS. This is a system which covers and manages the whole organization of the hospital [3].

\subsection{History}

Hospital Information System or HIS is a computerized system which is designed for management of hospital information such as administrative or medical information in order to assist the professional health jobs in their tasks effectively. For the first time, Hospital Information System was designed and developed in 1960, and it is considered as one of the most essential elements in management of hospital information and administrating hospital tasks, now. At first, systems included several central computers which were connected with each other through terminal ends. Today, they are networked by microcomputers and personal computers. Systems control patients' issues; they also manage needed hospital materials and their maintenance. Hospital Information System has unified and centralized all clinical, financial and management hospital requests; this is the reason why this system is called Hospital Data Processing system [4].

\section{Methods}

For transferring data among urology research centers unified systems are needed. Of course, the methods which were applied in this study are clarified by defining the methods which can be used in data transfer. These definitions are as follows.

\subsection{Health Information System}

It is a system which collects, handles, saves, and analyzes and then publishes the achieved information. HIS is a coherent information system for management, saving and reviewing patients' information. Information System collects, processes, analyzes and publishes the data based on a specified aim [4].

\subsection{General Structure of Information System}

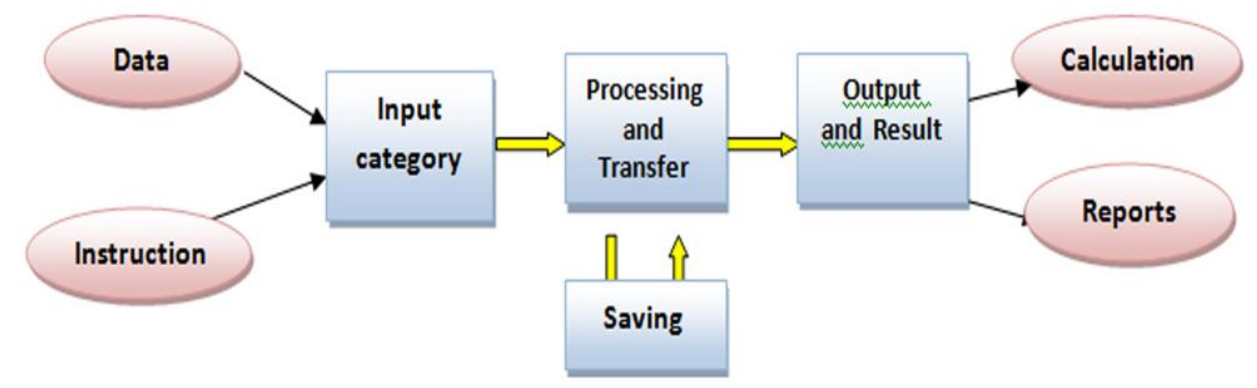

Figure 1. General Structure of Information System

\subsection{Administrative Automation}

Administrative Automation includes all official and non-official electronic systems; it is related to information exchange between individuals in and out of institute and vice versa. The only thing which differentiates administrative automation from data processing, data management system and decision support system is 'communication'. The task of administrative automation is to facilitate all kinds of communications in both forms, oral and written. These systems support the administrative units by the use of information technology and endow more efficiency to the offices [5]. 
In addition to architecture and technology used in development process regarding operating equipments, administrative automated systems enjoy various potentials.

If administrative automation's definition supports communication in an organization generally, it can be pointed out that the least capabilities are as follows:

1. Supporting all kinds of organizational and intra-organizational correspondences and communications electronically ;

2. Possibility of sharing tasks, organizational information and the ability to pursue the affairs with the goal of achieving group and team works;

3. The possibility of presenting organizational forms electronically;

4. Work flow engine for achieving organizational procedures;

5. Making benefit from electronic document management system in order to support all kinds of organizational documents;

6. Making benefit from organizational central record system;

7. Supporting centralized and distributed organizations;

8. Possibility of information transfer with other administrative automated systems;

9. Possibility of information exchange with outside the organization through different kinds of communicative data standards such as email, fax and SMS[5].

\subsection{System Boundary}

Research Information System which is called administrative automated system is a system which works as a link among research centers and universities of medical sciences and stem cells organization. Research centers are linked to the system just by automation. This system is the linking system among urology research centers and universities; on the other hand the connection between these centers with other related organs such as stem cells organization and other research centers are provided indirectly. Other organizations can also establish a direct communication with Administrative Automated System; this system should define special boundary in the field of urology with universities, stem cells organizations, urology research centers (dependent and independent) and their operators [5].

\subsection{System Operators}

Researcher, referee, manager of center, website manager, research expert, laboratory manager, laboratory expert, coordinator of research centers, supervisor, coordinator and website administrator of stem cells centers, technical leaders of system (in the system admins who are considered as virtual leaders of organization enjoy the best access; they are also supporters and initiators of automation, and have a large number of operators) [6].

\subsection{Transfer Criteria}

Along development of information technology and its influence on the society, information standardization and classification for sharing affairs seem important. Hospitals are considered as important constitutions which give health and medical services; they have equipments which play significant roles in society's physical and psychological health, also in researches and teaching experts of health and medical sections. Thus, computer plays an important role here, as much as in other sciences. Computer is applied in various areas while it is used for control and treatment in order to economize on the costs (saving time, preventing repetition, and attention). Usage of computer in hospitals enjoys a lot of merits in documenting patients' records, registering vital signs, surgeries, emergency cares, nursing specialized systems, laboratories, in doing radiology, pathology; moreover, its application traces are seen in financial affairs, storage, insurance, supply department and accounting [7]. 


\section{Results}

In this Section, it is declared that the study found out that HL7 can be considered as a proper system for data transfer among urology centers.

\subsection{HL7 and Its Role on Health Information System}

Health Level Seven is an international standard in information transfer in medical sciences. HL7 is not software, but it is a firm standard in 'e-Health' area according to which great medical sciences software especially HIS, today should be designed and implemented. Now, producer of Hospital or Health Information System know that any software which lacks this standard has no place among medical science technologies. With this introduction, more explanation is presented on this standard with the emphasis on the point that this issue needs more studies. HL7 is a private institution which works on promotion of health care standards. Special standards of this organization are described as V3 and V2X on its products. The aim of HL7 as an international association for health care is to establish collaboration between science experts and information researchers, also to define management information transfer standards and to establish a coherent electronic health record. HL7 facilitates usage of common standards in and out of health care organizations to promote effectiveness and efficiency of health services [8].

\subsection{Organizational Strategy HL7}

The central and main HL7 agency is located in Michigan. HL7 is an organizational institute which is confirmed and validated by ANSI (American National Standard Institute). HL7 first appeared in 1987 for defining a standard for HIS. HL7 provided a standard structure for unification, sharing, marketing electronic health information and transfer. It supports clinical surgery standards, management standards, transfer standards, and evaluating health services which are common all over the world. Hospitals, for example, make use of various computer systems for different purposes from presenting bills to pursuing the treatment. When one of these systems receives new data, all of them must be connected to start sharing. Hl7 is a language by which different health care systems can operate [9].

\section{- Words Classifications and Technical Words}

LOINC standard Usage: clinical and laboratory words and codes are in fact a coded system which is used in laboratories in evaluations, and in clinical documents; also they are applied in electronic transfer among individual electronic systems [4].

\section{- Compacting}

JPEG: It is considered as an image compacting standard and as an image converter to a series of 'bytes'. It is possible to use WAVELET; it is considered as an information extraction standard from images or sound waves which makes them prepared for compacting with wave fluctuation [4].

\section{- Confidentiality and Security}

TLS actually prepares the security for comprehensiveness and confidentiality between two applied programs which are connected to each other; this is extended to the issue of 'security of communications on the network'; also IP sec can be used for coding and identification [4]. 


\section{- Transfer}

In order to find an easy access to objects, also to access a mechanism for a structured transfer between two computers by language SOAP XML standard is used [4].

\section{- Hardware}

Any needed hardware such as equipments, computer physical elements, electronic components, circuits, mechanical needed elements for users and for the desired connection should be supplied by the designer based on number of operators [4].

\section{- Software}

The same systemic program should be taught to all operators of research center. Preparation of Information Technology for establishing a connection between two computers or more also establishing a connection among computer equipments is done by inner or outer communications based on similar protocols [4].

\section{Discussion}

Limitation of the study was to find access to available resources of information so in this study several interviews were done with well-known Iranian Professors, hospital researchers and administrators. In this section, the results regarding different data transfer methods are brought into discussion. The way the administrative automation can be applied in urology centers are discussed. Indeed, the effects of results seem to be clarification of the different data transfer style.

\section{- Implementation of Administrative Automation in Centers}

1. Installation and implementation of LAN; the personnel who are involved in correspondences should access to the mentioned network by ease;

2. Providing the proper bandwidth for connection with university through PTMT lines (on wide Backbone of university) or internet high speed lines, ADSL2+;

3. Defining a person as automation connector with predetermined criteria ;

4. Providing, installing and triggering the server (with the identification announced by Information and Communication Technology Center) for installation of control network softwares (ISA, ADC, DHCP, etc.);

5. Equipping the central secretariat with at least two computers, one scanner, printer according to identification announced by Information and Communication Technology Center;

6. Completing the hardware equipments in a way that all personnel involved in correspondences exchange can have an easy access to automation system;

7. Tree organizational chart including departments and offices' titles, job titles, individual's position, their first and last names (in Persian and in English) according to identification announced by Information and Communication Technology Center;

8. Announcing the center's preparation to be visited by the experts of Information and Communication Technology Center. Related checklists should be completed;

9. Programming and arranging a time schedule by the connector for training automation classes for personnel such as experts, office manager, registry officer, senior managers, etc., based on their access level to the system;

10. Providing a time schedule triggering program by the connector with the collaboration of Information and Communication Technology Center;

11. Establishing internal and external correspondence cycles; also editing the required processes in PILOT step;

12. Running the system and omission of physical correspondences [10]. 


\subsection{Resources Specification:}

Available Resources:

1. Financial Resources: They are determined by University of Medical Sciences.

2. Equipments: They include all the available physical equipments and facilities for triggering the mentioned system in the Urology Research Center.

3. Human Forces:

- Temporary Human Force: It includes human forces who have the duty of collecting, analysis, designation, implementation, and supporting of the Information System; this group includes programmer, system analyzer, and other team members.

- The Resource Provider: The resources are provided by University of Medical Sciences; this university is in charge with research centers' affairs [11].

At first an estimation of the system's cost should be done.

\subsection{Cost Estimation Method:}

Activity-Based Costing: Activity-based costing is a method for measuring some items such as evaluating costs, activity efficiency, resources, etc.

It means the connection between resources and activities and then activities and costs.

Accordingly, costs include the following items:

- Measuring efficiency of works' processes, activity by activity;

- Estimation of system's final cost according to the available resources' costs;

- Identification of opportunities in order to promote system's efficiency.

After presenting the cost estimation of establishing an information system to University of Medical Sciences, it is the university that should supply the researchers with the resources;

- Specification: It is done based on the instructions determined by University of Medical Sciences and research centers;

- Classification of levels: Resources levels are determined based on priorities in programming stage; these priorities are also arranged regarding approved research plans.

- Application: Resources application method is defined by manager of the system regarding his authorities; research experts also have the duty to act according to management budget policies approved for using the resources and regarding the assumptions and limitations [12].

\subsection{Administrative Criteria:}

Approval Process of Research Plans: 1. Registering on the website through 'register and send your research ideas', receiving user name and password; entering to operator's control panel;

2. Completing form of 'research idea registry':

- Sending the research idea registry form to the center through website of the center;

- Sending the research idea to the research committee of the center; the idea will be judged there;

- The research idea is put into discussion in research committee to achieve the approval;

- If the research idea is approved, the proposal should be prepared in a determined time according to the granted permission [13].

3. Writing a research proposal:

- Defining one of the faculty members of University of Medical Sciences as the major research plan executer (this selection is done according to regulation of University Undersecretary for Research); it should be mentioned that all rights regarding the plan are arranged in the center through a memorandum of agreement with the major 
executer; the rights can belong to the author. Also, if both sides agree, selection of the major executer can be done superficially;

- Completing center's series of forms, memorandum of agreement form, commitment form, etc. (First, forms should be downloaded from information part, then they should be completed and should be sent by control panel after scanning);

- Announcing the approval view of the center in order to arrange the proposal in a determined period;

- Providing the proposal from the website of Research Information System;

- Following the status of the plan according to Research Information System website (all the medical sciences research plans should be approved by the Research Information System website; it is not acceptable if the processes are done personally.);

4. Providing research proposal through Research Information System website:

- Registering and receiving username and password;

- Entering to the Research Information System website;

- Defining an icon for the research plan;

- Choosing a plan which is done not as a dissertation.

In order to approve a research plan, some processes should be pursued; they are as follows:

Receiving the proposal from the executer; then referring to the Undersecretary and checking if it is adapted to the center's priority regarding its contents. Afterwards, the executer should be informed to hand in the completed form of proposal in a two-week period. A referee should be determined; a two-week period is needed to consider their view points. Then, referees' opinions are delivered; these opinions are handed in to executers; meanwhile reply of complementary plan executer is received. Next, it is reported to the research committee, and now it is this committee who accepts or rejects the plan. Sometimes, the research plan is approved under special conditions. If it is rejected, the executer will be informed. It is sent to moral committee if accepted. And if it is rejected in this part, the executer is informed. A contract is arranged for an accepted proposal. But if the proposal is accepted under certain conditions, first, those conditions should be fulfilled [14].

\subsection{HIS Merits in Hospital Processes}

- Promotion of efficiency level;

- Making benefit from computer technologies;

- Omission of repeated and unnecessary procedures;

- Time management (reducing admission time, also reducing discharge time, time of transferring patients, physicians' ORDER writing, paraclinical requests, the time of obtaining the answers, the time of referring to previous records data);

- Increasing the amount of attention in recording the information and requests which are written in an illegible handwriting;

- Facilitating the communication among intra sections and promoting patients' contentment;

- Giving better services, registering the statistics, daily and time-scheduled reports;

- Being informed of hospital income status and the costs;

- Bringing balance to human force;

- Qualitative promotion of medical care;

- Extraction of statistics and information by smart methods;

- Connecting to medical systems;

- Achieving a distributed information bank along the entire country and establishing a connection with the universal health networks [15]. 


\section{Conclusions}

Without Information Technology, today's physicians are not even at the same knowledge level as the past. With the all-dimensional new life methods, information technology plays a significant role in treatment and medical researches. It is possible to define life in a special direction; this is done by information technology since it thoroughly improves life quality. Nowadays, by owning just a record code, it is possible to access the whole medical information. Any information related to physical or psychological health or a patient's status which is recorded on electronic systems are used for transfer, saving, receiving, reviewing, connecting and exchanging multimedia data in order to get ready for primary health care and heath services.

Health Information System includes all health and therapeutic information related to the health of an individual during his life time; it is established in therapeutic health system and is maintained confidentially. In order to give the services to patients, this system should always be available to legitimate service providers. Health Information System is a secure electronic information system which is available to service providers located at therapeutic health centers; it contains the previous records meanwhile new treatment information can be added to it [16].

It is important in promoting clinical care and decreasing medical mistakes to have an access to patients' information. Health Information System is a coherent collection of health care information of an individual gathered in a long term which is applicable for all service providers in any time and any center. Any of individuals' related health care systems are considered as parts of a unified Health information System. Those systems which belong to a health information system enjoy this capability meanwhile they contribute to the development of patient's health level; they also save hundreds of time and reduce the care costs. An available health information system brings a better decision making in the treatment processes and develops therapeutic results. Clear data is collected for public health and researches. But this development is just achieved at a time when a standard method is selected. So, it seems necessary that all medical centers around the world make advantage of information technology in managing and control data transfer, and enjoy its benefits.

\section{Acknowledgements}

We are grateful to Dr. Tofighi and Dr. Arezou Taheri. Thanks to collaboration of Dr. Neekfarjam (arrangement administrator of Urology Center of Children's Medical Center), Dr. Mortazavi, the head of Urology and Kidney Research Center in Isfahan University, and Dr. Hamidi, urological surgeon, researcher and member of Sina Urology Hospital.

\section{References}

[1] "Health Information System", available at http://vcr.tums.ac.ir/content/?contentID=46 (accessed January $\left.5^{\text {th }}, 2015\right)$.

[2] Hospital Activities Support: an Interview with Dr. Neekfarjam (Coordinator of Urology Center of Children's Medical Center).

[3] “HIS Goal”, available at http://rco.tums.ac.ir/Portfolio.aspx(accessed March 10 $\left.0^{\text {th }}, 2015\right)$

[4] "Health Information System", available at http://research.tums.ac.ir/informatics.phtml (accessed January 5th, 2015)

[5] “Administrative Automation", available at http://vo.tums.ac.ir/http://vo.tums.ac.ir/(accessed January 5 $5^{\text {th }}$, 2015)

[6] "System Operators: an Interview with Mahzaad Rousta", Coordinator of Urology Research Center, Isfahan.

[7] "Transfer Criteria", available at http://beheshti.umsha.ac.ir/index.aspx?siteid=9\& pageid=6487 (accessed August $24^{\text {th }}, 2015$ )

[8] "HL7 and Its Role in Health Information System: an Interview with Dr. Hamidi", Urology Surgeon and Researcher, Member of Urology Research Center, Sina Hospital.

[9] "Organizational Strategy of HL7", available at http://www.gums.ac.ir/urc/default-530.aspx(accessed January $5^{\text {th }}, 2015$ ) 
[10] "Administrative Automation settling Process in Centers", available at http://imhr.tums.ac.ir/(accessed January $5^{\text {th }}, 2015$ )

[11] "Resources Designations: an interview with Technical Leaders of Tehran University of Medical Sciences", Virtual Organization: Maryam Ahmadi, Fateme Baatmanghilich, Samira Elmi, Fateme Golmahi, Samira Mortezaee.

[12] "Resource Specifications Method: an interview with Dr. Mortazavai", Boss of Urology and Renal Research Center, Isfahan University.

[13] Supplying Research Proposal from Research Information Website: Administrator of Urology Research Center, Rasht.

[14] "HIS Merits in Hospital Procedures: an interview with Dr. Kordi", Researcher, Referee of Tehran University: Research Center.

[15] "Role of Information Technology in Treatment and in Medical Surveys: an interview with Office Administrator", Dr. Pourmand, Sina Hospital 13.Research papers Approval procedures: Vafaee, Administrator of Urology and Nephrology Center in Shahid Beheshti Hospital, Hamedan.

[16] "The Role of Access to Patients' Information on Improvement of Clinical Care: an interview with Mo'taghed", Engineer and Supporter of System in Urology Research Center, Hamedan.
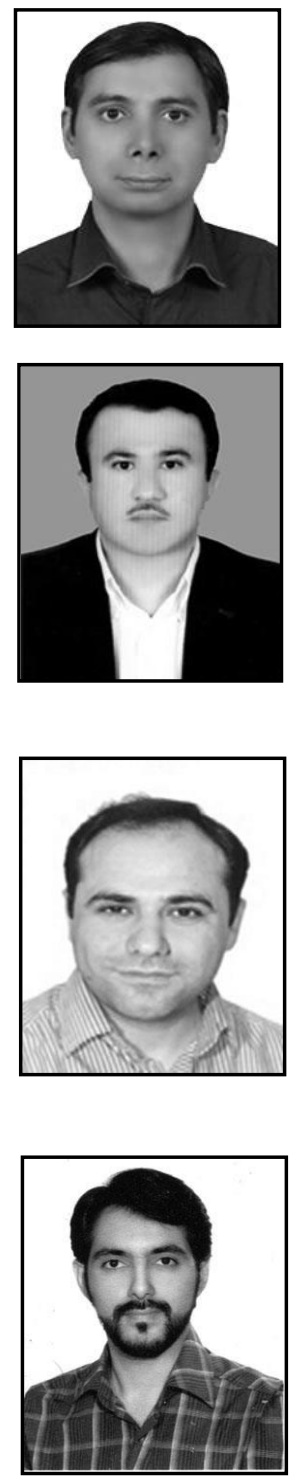

\section{Authors}

Abdolhossein Ayoubi, (1983), Jahrom, Iran. M.Sc. of Medical Informatics Technology in Biomedical Engineering Department, Amirkabir University of Technology, Tehran, Iran. Working as a specialist of Medical Informatics Technology. I have published 8 books in Createspace/Amazon Publication and one other book in Atraa publication, Iran, and several papers in Biomedical Engineering.

Mohammad Sadegh Sanie, (1977) Jahrom, Iran. MD in Anesthesia and Critical Care, a member of Scientific Board in Anesthesia and Critical Care in Jahrom University of Medical Sciences. I collaborate with two journals as their reviewer which include Anesthesia and Critical Care ( AACC) and (Pars) Research Periodical, I have published 2 books in Createspace/Amazon Publication, and several papers in conferences. Sadegh_532@yahoo.com, Tel:00989177002599

Moharram Kazemi, (1972), Khoy, Iran. M. Sc. of Medical Informatics Technology, Amir Kabir Technical University, Biomedical department, Tehran, Iran. Working as a cardiac Electrophysiology Clinical specialist, I published 2 books via Createspace/Amazon publication, and several papers in Biomedical engineering. Email Address: kazemi1az@yahoo.com, Tel: 0098121366802, Address: plaque:60 ,Third Floor, $39^{\text {th }}$ Gisha St, Tehran, Iran. postal code 1447975547

Saman Sobh Heydari, (1976), Tabriz, Iran. M.Sc. of Medical Informatics Technology, Biomedical Engineering Department, Amirkabir University of Technology, Tehran, Iran; I published one book via Createspace/Amazon publication, and several papers in Biomedical engineering field, working as a specialist of Medical Informatics Technology. Email Address: saman303@yahoo.com; Tel: 00989166433448. Address: Plaque: 42, 2nd Baharan, Baharan Neighbor, Dezfoul, Khoozestan Province, Iran, Postal Code: 6461687653 
International Journal of Bio-Science and Bio-Technology

Vol.8, No.1 (2016) 